\title{
Truncated E-Cadherin Potentiates Cell Death in Prostate Epithelial Cells
}

\author{
Jonathan Rios-Doria ${ }^{1,2}$ and Mark L. Day ${ }^{1,2,3 *}$ \\ 'Department of Urology, University of Michigan, Ann Arbor, Michigan \\ ${ }^{2}$ Program in Cellular and Molecular Biology, University of Michigan, Ann Arbor, Michigan \\ ${ }^{3}$ University of Michigan Comprehensive Cancer Center, University of Michigan, Ann Arbor, Michigan
}

\begin{abstract}
BACKGROUND. E-cadherin, a fundamental component of the adherens junction, is known to mediate aggregation-dependent cell survival. We have previously identified a novel, calpaindependent proteolytic cleavage of E-cadherin that resulted in the generation of a stable 100-kDa E-cadherin fragment $\left(\mathrm{E}-\mathrm{cad}^{100}\right)$ in prostate epithelial cells in response to cell death stimuli. We postulated that the E-cad ${ }^{100}$ fragment may play a role in abrogating survival of LNCaP cells following induction of apoptosis.

METHODS. Wild-type E-cadherin and E-cad ${ }^{100}$ were engineered, tagged with GFP, and stably expressed in LNCaP cells. These cell lines were characterized for E-cadherin-GFP/ $\beta$-catenin interactions, endogenous E-cadherin and $\beta$-catenin expression, and sensitivity to apoptosis induced by PKC activation.

RESULTS. E-cad ${ }^{100}$-GFP demonstrated a punctuate expression pattern, in contrast to E-cad ${ }^{120}$-GFP, which was membrane-localized. E-cad ${ }^{100}$-GFP, unlike E-cad ${ }^{120}$-GFP, failed to bind to and co-localize with $\beta$-catenin. Transient or stable overexpression of E-cad ${ }^{100}$ resulted in the downregulation of endogenous E-cadherin expression at the cell membrane. Activation of PKC in LNCaP cells which overexpressed E-cad ${ }^{100}$ potentiated cell death.

CONCLUSIONS. Truncated E-cadherin may play a role in the regulation of endogenous E-cadherin expression and epithelial cell survival. Prostate 63: 259-268, 2005.
\end{abstract}

(C) 2004 Wiley-Liss, Inc.

KEY WORDS: $\quad$ LNCaP; apoptosis; calpain; $\beta$-catenin; PKC

\section{INTRODUCTION}

E-cadherin is a transmembrane glycoprotein that functions in the formation of adherens junctions in epithelial cells. E-cadherin mediates calcium-dependent, homotypic adhesion between like molecules on adjacent cells. The structure of E-cadherin consists of five extracellular domains, a transmembrane domain, and a cytoplasmic domain [1]. In the cytoplasmic domain, E-cadherin can bind to either $\beta$ - or $\gamma$-catenin, which in turn bind to $\alpha$-catenin and links E-cadherin to the actin cytoskeleton. It has been demonstrated that the $\beta$-catenin binding domain of E-cadherin is critical for E-cadherin adhesive function [2,3].

Other than mediating inter-cellular adhesion, E-cadherin can also mediate intracellular signaling and has been shown to play a role in cell survival. It has been shown that E-cadherin plays a role in anchorageindependent growth and survival of squamous carci- noma cells and can suppress apoptosis of immortalized granulosa cells $[4,5]$. N-cadherin can also mediate cell survival in several cell types [6-9]. Our laboratory demonstrated E-cadherin-mediated aggregationdependent survival through the retinoblastoma cell cycle control pathway when prostate epithelial cells are

\footnotetext{
Abbreviations: TPA, 12-O-tetradecanoylphorbol-13-acetate; PKC protein kinase C; GFP, green fluorescent protein; $\mathrm{E}-\mathrm{cad}^{100}, \mathrm{M}_{\mathrm{r}}$ 100,000 E-cadherin; E-cad ${ }^{120}, \mathrm{M}_{\mathrm{r}} 120,000$ E-cadherin.

Grant sponsor: National Institutes of Health; Grant number: RO1 DK56137.

*Correspondence to: Mark L. Day, Department of Urology, University of Michigan, Room 6219 CCGC, 1500 East Medical Center Drive, Ann Arbor, MI 48109-0944. E-mail: mday@umich.edu. Received 15 July 2004; Accepted 17 August 2004 DOI 10.1002/pros.20179

Published online 10 November 2004 in Wiley InterScience (www.interscience.wiley.com).
} 
challenged with cell death stimuli from PKC activation [10]. Activation of PKC in LNCaP cells resulted in both a viable and apoptotic cell population. Cells which were able to aggregate and become contact-inhibited were found to be viable and contained functional E-cadherin [10]. Examination of the non-aggregated cell population revealed the presence of highly stable 97- and 100-kDa E-cadherin proteolytic cleavage fragments, which lacked the $\beta$-catenin binding domain $[11,12]$. Recently, we have found that the 100-kDafragment $\left(\mathrm{E}-\mathrm{cad}^{100}\right)$ was generated by calpain protease in response to PKC activation, as well as calcium ionophore treatment [12]. The role of this cleavage fragment in the cell survival of prostate epithelial cells has not been investigated.

E-cadherin proteolysis was first identified by the discovery of a soluble 80-kDa extracellular fragment of E-cadherin that was able to disrupt cell-cell adhesion [13]. This soluble form of E-cadherin is likely generated by stromelysin- 1 and matrilysin cleavage [14]. E-cadherin has also been shown to be targeted by caspases during apoptosis [15]. Our laboratory has recently shown that calpain protease mediates the cleavage of E-cadherin to generate a stable $100-\mathrm{kDa}$ fragment (E-cad $\left.{ }^{100}\right)$, which is slightly larger than the $80-\mathrm{kDa}$ fragment. We postulated that the generation of a $\beta$-catenin-binding deficient fragment of E-cadherin may have specific functional consequences in cell survival. However, the function of a defined proteolytic cleavage product of E-cadherin has yet to be uncovered.

Calpain can participate in apoptotic programs in some cell types, although the precise role of calpain in apoptosis remains to be elucidated [16]. Recent studies indicate that there may be a crosstalk between the calpain and caspase proteolytic systems [17-19]. We have previously shown that calpain can rapidly generate E-cad ${ }^{100}$ in response to PKC activation or calcium ionophore, which are known inducers of apoptosis in LNCaP cells $[12,20,21]$. It is postulated therefore, that E-cad ${ }^{100}$ may play a role in the apoptotic cascade in these cells by actively disrupting aggregation-dependent survival. We investigated E-cad ${ }^{100}$ overexpression in LNCaP prostate epithelial cells and its potential role in cell survival.

\section{MATERIALS AND METHODS}

\section{Tissue Culture Cell Lines and Transfections}

The LNCaP, MCF-7 and SKBR3 cell lines were grown in $7.5 \%$ FBS supplemented with $2 \mathrm{mM}$ L-glutamine, 100 units $/ \mathrm{ml}$ penicillin, and $100 \mu \mathrm{g} / \mathrm{ml}$ streptomycin. LNCaP stable cell lines were supplemented with $600 \mu \mathrm{g} / \mathrm{ml}$ G418 (Bio Whittaker). Cells were incubated at $37^{\circ} \mathrm{C}$ and subcultured weekly. Transfection of LNCaP cells was performed using DMRIE-C reagent according to the manufacturer's protocol (Invitrogen). The population of cells which had the strongest expression of GFP was selected using fluorescence activated cell sorting (FACS) (University of Michigan Flow Cytometry Core). Briefly, the top $10 \%$ of cells expressing GFP were collected following transfection and were re-plated in media supplemented with G418 to generate stable cell lines. TPA was used at a final concentration of $10 \mathrm{nM}$ (Alexis Biochemicals).

\section{Inserting Stop Codons}

E-cadherin stop mutants were generated by inserting stop codons in the cDNA sequence of a wild-type E-cadherin plasmid [12]. To generate N809Stop, the codon which coded for an asparigine at amino acid 809 was replaced with a stop codon in the cDNA sequence of a wild-type E-cadherin plasmid template [12]. The following primers were used in the Quikchange kit (Stratagene) to generate the N809Stop mutant plasmid, forward: 5' CCG ATG AAA TTG GAT AGT TTA TTG ATG $3^{\prime}$; reverse: 5'CAT CAA TAA ACT ATC CAA TTT CAT CGG $3^{\prime}$. Stop codons were also inserted after codons R784, Y797, and P801. Primers that were used to generate these truncation mutants will be provided upon request.

To generate the E-cad ${ }^{100}$-GFP construct, a Sac II site was inserted after the glycine in the amino acid sequence DEIGN ${ }^{809}$ in the cDNA sequence of the same wild-type E-cadherin plasmid template [12]. This was the same location that the stop codon was inserted to generate N809Stop. The primers that were used to insert the SacII site were as follows: forward $5^{\prime}$ CCG ATG AAA TTG GAC CGC GGA ATT TTA TTG 3'; reverse $5^{\prime}$ CAA TAA AAT TCC GCG GTC CAA TTT CAT CGG $3^{\prime}$. The $5^{\prime}$ HindIII/SacII $3^{\prime}$ fragment was then ligated into the $\mathrm{pEGFP-N1}$ vector (Clontech). To create full-length E-cad ${ }^{120}$-GFP, a Sac II site was inserted after the last codon in a wild-type E-cadherin plasmid [12]. This fragment was then ligated into the pEGFP-N1 vector.

\section{Immunoprecipitation}

Protein lysates were prepared from cultured cells in the following buffer: $50 \mathrm{mM}$ Tris, pH 7.5, $120 \mathrm{mM} \mathrm{NaCl}$, $0.5 \%$ Nonidet $\mathrm{p}-40,40 \mu \mathrm{M}$ phenylmethylsulfonyl fluoride (PMSF), $50 \mu \mathrm{g} / \mathrm{ml}$ leupeptin, $50 \mu \mathrm{g} / \mathrm{ml}$ aprotinin, $200 \mu \mathrm{M}$ orthovanadate, and $1 \mathrm{mM}$ EGTA. Cells were allowed to lyse $1 \mathrm{hr}$ on ice, centrifuged, supernatants extracted, and quantitated using a Bradford assay. Lysates were separated by $6 \%$ NOVEX gels and analyzed using chemiluminescence (Amersham Pharmacia Biotech). For isolation of the cell membrane/ organelle fraction, the ProteoExtract kit was used (Calbiochem). 
For immunoprecipitations, cell lysates were prepared and quantitated as described above. The lysates were pre-cleared for $30 \mathrm{~min}$ at $4{ }^{\circ} \mathrm{C}$ with protein-A sepharose beads (Zymed Laboratories). All beads were diluted and mixed for $1 \mathrm{hr}$ at room temperature with an equal volume of TBST (50 mM Tris, $\mathrm{pH} 7.5,120 \mathrm{mM}$ $\mathrm{NaCl}, 2 \%$ Tween 20$)+2.5 \%$ milk prior to use. Following pre-clearing, lysates were aliquotted into microcentrifuge tubes, where an equal volume of lysate was used for each immunoprecipitation reaction. The volumes of each reaction were equalized to $500 \mu \mathrm{l}$ with lysis buffer, $5 \mu \mathrm{g}$ of primary antibody was added, and the reaction was carried out at $4^{\circ} \mathrm{C}$ rotating endover-end. The following day, the beads were added and the reaction was allowed to continue to rotate end-over-end at $4^{\circ} \mathrm{C}$ for $90 \mathrm{~min}$. The beads were then pelleted and washed three times with lysis buffer (+protease inhibitors). Reducing sample buffer was added to each pellet, boiled for $5 \mathrm{~min}$, centrifuged, and the supernatants loaded onto 6\% NOVEX Tris/Gly (Invitrogen) gels for Western analysis.

The antibodies used in immunoprecipitation and Western blot analysis were E-cadherin (HECD-1, Zymed), $\beta$-catenin (C2206, Sigma), GFP (MAB3580, Chemicon), and hsp-70 (MAB3516, Chemicon).

\section{Immunofluorescence Staining}

Chamber slides were coated with $5 \mu \mathrm{g} / \mathrm{ml}$ Fibronectin (F-1141, Sigma) in PBS overnight prior to cell plating. $3.0 \times 10^{5} \mathrm{LNCaP}$ stable cell lines were plated and grown for 2 days. Cells were fixed with $2 \%$ formaldehyde followed by 100\% ethanol, each for $5 \mathrm{~min}$ on ice. Cells were washed in PBS and blocked with TBST $+10 \%$ non-fat milk $+1.5 \%$ heat-inactivated FBS for $30 \mathrm{~min}$ at room temperature. Anti- $\beta$-catenin (Sigma) or anti-E-cadherin antibody was added (SC-1499, Santa Cruz) for $50 \mathrm{~min}$ at room temperature. After washing in blocking solution, cells were incubated with rhodamine-conjugated anti-goat or anti-rat secondary antibody for $1 \mathrm{hr}$ at room temperature (Santa Cruz). Cells were washed and visualized with an Olympus inverted fluorescent microscope. For immunostaining of transiently transfected cells, $2.0 \times 10^{5}$ MCF-7 cells were plated and grown for $24 \mathrm{hr}$. They were then transfected with E-cad ${ }^{100}$-GFP using the FuGene 6 reagent for 48 additional hr. Cells were then fixed and stained with anti-E-cadherin antibody as described above.

\section{Cell Viability Assay}

$1.5 \times 10^{5}$ cells were plated in 6-well dishes. Cells were treated with TPA when they reached $50 \%$ confluence. At time 0 and at $24 \mathrm{hr}$ post-treatment, the attached cells which stained with trypan blue were counted (Gibco).
Statistical analyses were performed using a Student's paired $t$-test with a two-tailed distribution. $P<0.05$ was considered statistically significant.

\section{Densitometry}

Densitometric analysis was performed using the Scion Image software. The ratio of band intensity between endogenous E-cadherin and Hsp-70 in the LNCaP E-cad ${ }^{100}$-GFP cell line was compared to the ratio in the LNCaP vector cell line, which was normalized to one.

\section{RESULTS}

\section{TPA Treatment Induces LNCaP Cell-Death and Generation of E-cad ${ }^{100}$}

Our laboratory has previously identified a 100-kDa E-cadherin fragment $\left(\mathrm{E}-\mathrm{cad}^{100}\right)$ that was generated by calpain in prostate and mammary epithelial cells in response to PKC activation or calcium influx [12]. PKC activation by treatment of cells with 12-Otetradecanoylphorbol-13-acetate (TPA) resulted in cellular aggregation of which the aggregated cells remained viable (Fig. 1A). However, a substantial proportion of cells were dissociated and rapidly underwent apoptosis (Fig. 1A) [10,20]. The viability of the TPA-treated cell population was significantly less $(70 \%)$ than untreated cells (88\%) (Fig. 1A). We have previously shown that aggregation-dependent survival of LNCaP cells required E-cadherin [10]. We hypothesized that PKC-induced apoptosis was associated with the disruption of the E-cadherin adhesion complex. Treatment of LNCaP cells with TPA resulted in the generation of E-cad ${ }^{100}$, which was previously shown to be mediated by calpain (Fig. 1B) [12]. Previously, we have shown that disruption of E-cadherinmediated adhesion using an E-cadherin blocking antibody potentiated TPA-induced apoptosis of LNCaP cells [10]. Furthermore, it has been shown that the $80-\mathrm{kDa}$ E-cadherin fragment also disrupts cell adhesion [13]. Based on these studies and our results, we hypothesized that E-cad ${ }^{100}$ might be affecting cell survival of LNCaP cells.

\section{Localization of E-cad ${ }^{100}$ Cleavage Site}

To investigate the role of E-cad ${ }^{100}$, we mapped the calpain cleavage site in order to engineer an E-cad ${ }^{100}$ construct. An E-cadherin antibody that maps to the extracellular domain of E-cadherin detected both fulllength E-cadherin and E-cad ${ }^{100}$ (Fig. 2A). In contrast, a C-terminal antibody to E-cadherin detected full-length E-cadherin but failed to detect E-cad ${ }^{100}$, suggesting that the calpain cleavage site resided in the cytoplasmic domain of E-cadherin (Fig. 2A). In order to ascertain 
A

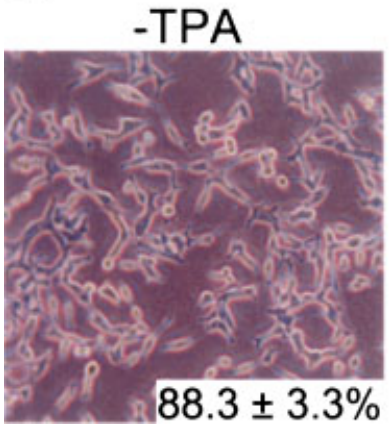

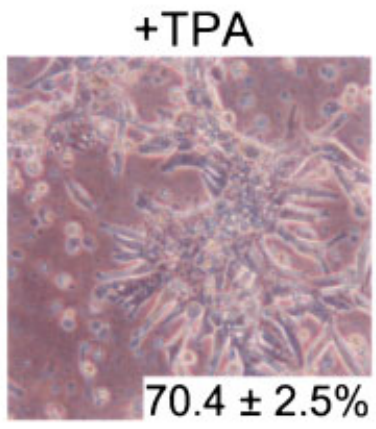

B

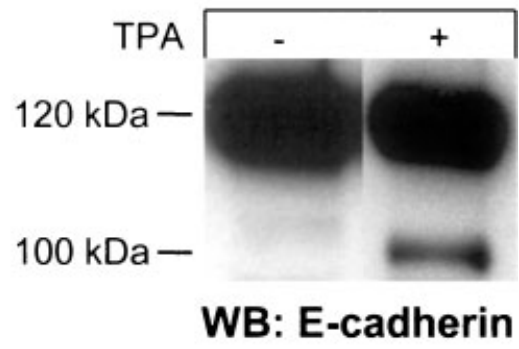

Fig. I. TPA treatment induces LNCaP cell-death and generation of E-cad ${ }^{100}$. A: Untreated and 24 hrTPA-treated LNCaP cells were visualized by phase contrast microscopy. Cell viability was performed in duplicate. Magnification is at $20 \times$. B: Whole cell lysates of untreated or $24 \mathrm{hr}$ TPA-treated LNCaP cells were resolved by SDS-PAGE and immunoblotted with antibody to E-cadherin. [Color figure can be viewed in the online issue, which is available at www.interscience.wiley.com.]

the E-cad ${ }^{100}$ cleavage site more precisely, E-cadherin mutants were created in which stop codons were inserted in wild-type E-cadherin cDNA, resulting in a truncated E-cadherin protein at potential calpain cleavage sites (Fig. 2B). The calpain consensus site has been shown to contain a valine, leucine, or isoleucine at the P2 position, and a tyrosine, glycine, or arginine at the P1 position, though the amino acids at the P1 position can be varied [16]. Stop codons were inserted in the cDNA to replace the codon for the amino acid indicated (Fig. 2B). These E-cadherin truncation mutants were then transiently expressed in E-cad - /SKBR3 cells and immunoblotted with an antibody which recognizes the extracellular domain of $\mathrm{E}-$ cadherin (Fig. 2C). Alignment of the expressed Ecadherin truncation mutants with endogenous E-cad ${ }^{100}$ demonstrated that the truncation following the asparigine at amino acid 809 had an identical electrophoretic mobility to endogenous E-cad ${ }^{100}$. This particular mutant also contained a consensus calpain cleavage site consisting of an isoleucine at the P2 position, and a glycine at the P1 position [16]. This mutant was therefore chosen to represent exogenous E-cad ${ }^{100}$ in future experiments.

\section{Expression of E-cadherin/GFP Constructs in LNCaP Cells}

The E-cad ${ }^{100}$ fragment was cloned into a GFP vector, and stably expressed in LNCaP cells. LNCaP stable cell lines expressing a full-length, wild-type E-cadherinGFP construct, as well as the empty GFP vector were also created. High expression of these proteins in the cell lines was achieved by subjecting the transfected pools to FACS, in which the top $10 \%$ of fluorescing cells were collected and re-plated in selection media (data not shown). The cell lines were visualized using fluorescence microscopy to determine the level of expression of the expressed proteins. The LNCaP

vector cell line displayed whole cell staining of the GFP protein (Fig. 3A). As expected, the LNCaP E$\operatorname{cad}^{120}$-GFP cell line demonstrated strong E-cadherin staining at the cell membrane (Fig. 3A). In contrast,

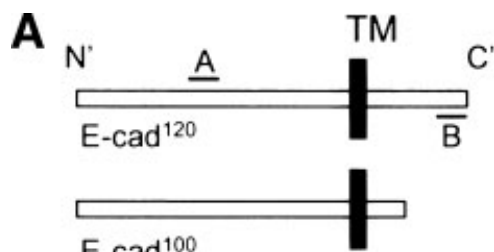

$E-\operatorname{cad}^{100}$

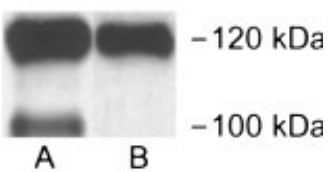

B
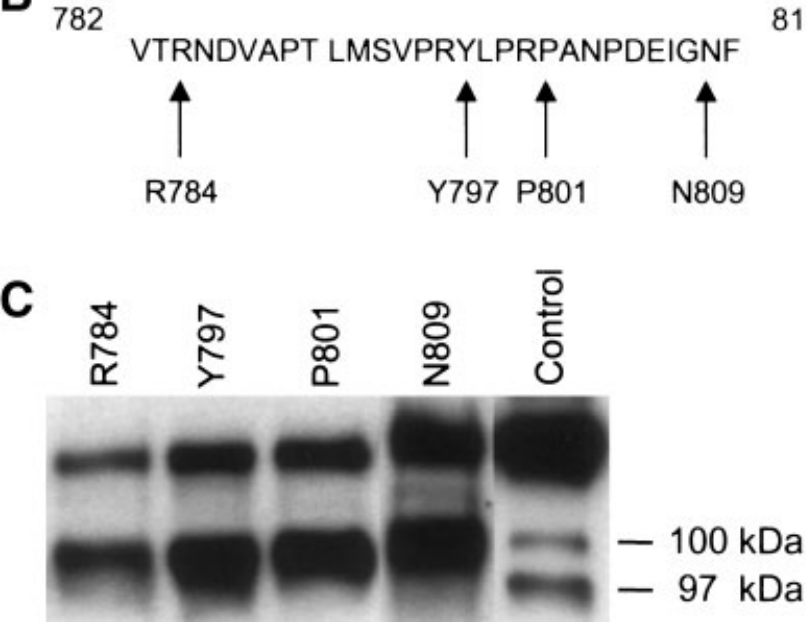

Fig. 2. Antibody mapping and construction of E-cad ${ }^{100}$. A: A schematic of full-length E-cadherin and E-cad ${ }^{100}$ is shown. An antibody to the extracellular domain (antibody A) detected $\mathrm{E}-\mathrm{cad}^{100}$ in contrast to the C-terminal antibody (antibody B), which did not detect $\mathrm{E}-\mathrm{cad}^{100}$. B: Codons which encoded for amino acids that followed potential calpain cleavage sites were replaced with stop codons in the cDNA of a wild-type E-cadherin plasmid. C: Stop mutants were transiently transfected in SKBR3 cells and whole cell extracts were resolved by SDS-PAGE followed by immunoblotting with an E-cadherin antibody. The control lane represents cell lysate fromTPA-treated $\mathrm{LNCaP}$ cells. 

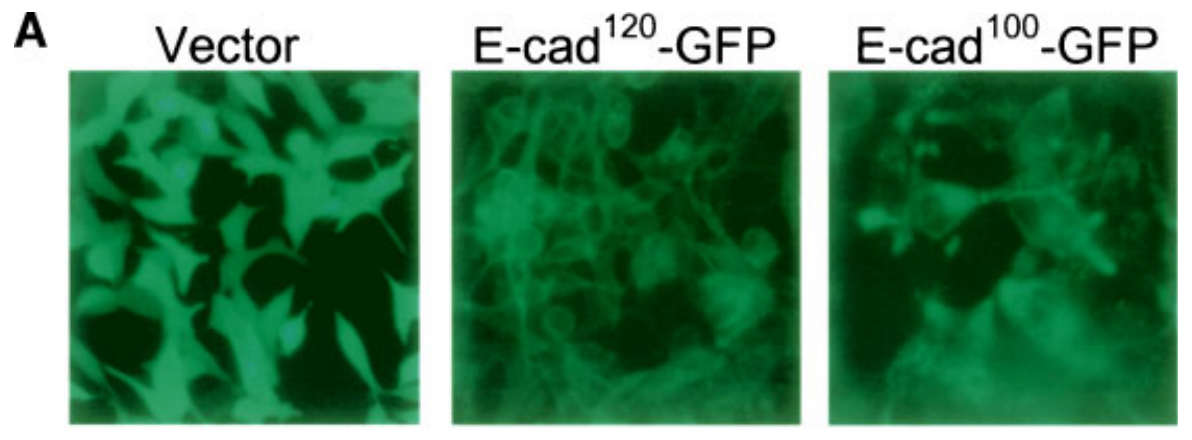

B
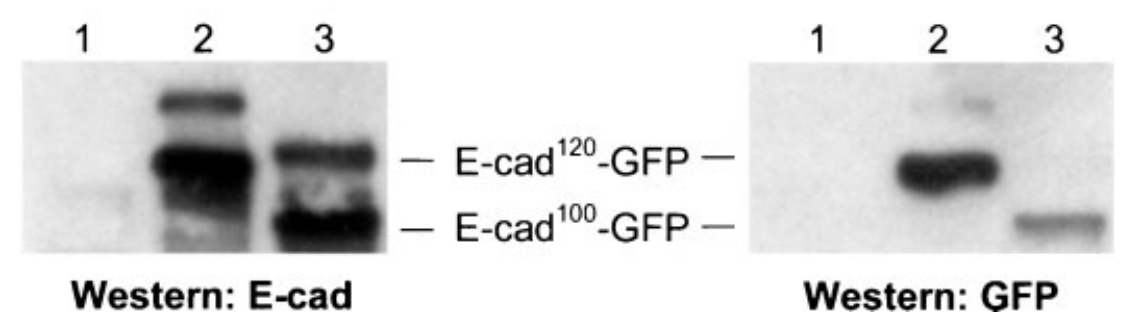

Fig. 3. E-cadherin-GFP expression in LNCaP cells. A:The LNCaP vector control, LNCaP E-cad ${ }^{120}-G F P$, and LNCaP E-cad ${ }^{100}-$ GFP cell lines were visualized by fluorescent microscopy. Magnification is at $20 \times$. B: Whole cell lysates from LNCaP vector cells (lane I), LNCaPE-cad ${ }^{120}-$ GFP cells (lane2), and LNCaPE-cad ${ }^{100}$-GFP(lane3) were resolved by SDS-PAGE and immunoblotted with an E-cadherin antibody (left panel) and a GFP antibody (right panel). [Color figure can be viewed in the online issue, which is available at www.interscience.wiley.com.]

E-cad ${ }^{100}$-GFP cell line demonstrated punctuate expression, which seemed to be localized to both the membrane and cytoplasm (Fig. 3A). Additionally, these cells displayed a more flattened phenotype than the control cell lines.

Western blot analysis demonstrated that the E-cad ${ }^{120}$ GFP and the E-cad ${ }^{100}$-GFP fusion proteins, which have molecular weights of $150-$ and $130-\mathrm{kDa}$, respectively, were stably expressed in the transfected cell lines (Fig. 3B). These proteins were detected with both antiE-cadherin and anti-GFP antibodies (Fig. 3B). To test whether the E-cad ${ }^{100}$-GFP protein associated with $\beta$ catenin, a co-immunoprecipitation assay was performed (Fig. 4A). Co-immunoprecipitation with a GFP-antibody demonstrated that E-cad ${ }^{120}$-GFP bound strongly to $\beta$-catenin, while E-cad ${ }^{100}$-GFP did not exhibit binding, confirming that this calpain-generated fragment has lost the $\beta$-catenin binding domain (Fig. 4A). The reciprocal experiment demonstrated that E-cad ${ }^{120}$-GFP, but not E-cad ${ }^{100}$-GFP could be coimmunoprecipitated with a $\beta$-catenin antibody (Fig. 4B). These results demonstrated that E-cad ${ }^{100}$-GFP did not possess the $\beta$-catenin binding domain.

\section{E-cad ${ }^{100}$-GFP Does Not Localize With $\beta$-Catenin}

We next investigated whether the GFP-tagged Ecadherin proteins co-localized with endogenous $\beta$-catenin. The LNCaP E-cad ${ }^{120}$-GFP and LNCaP E-cad ${ }^{100}$-GFP cell lines were stained for $\beta$-catenin and examined using immunofluorescence microscopy. E-cad ${ }^{120}$-GFP was localized primarily at the membrane, and exhibited strong junctional staining (Fig. 5A). $\beta$-catenin was also localized primarily at the membrane and an overlay of the images demonstrated strong co-localization of E-cad ${ }^{120}$-GFP and endogenous $\beta$-catenin (Fig. 5C

A

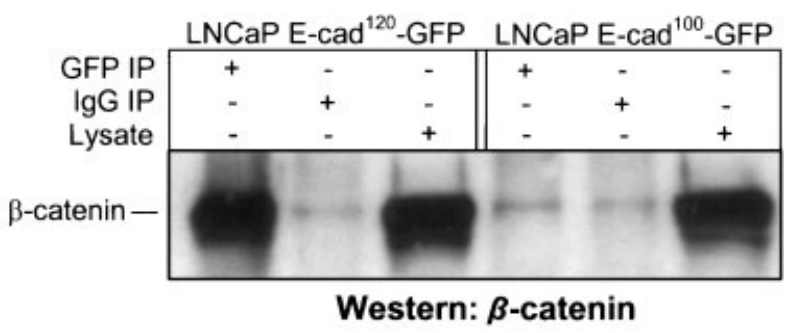

B

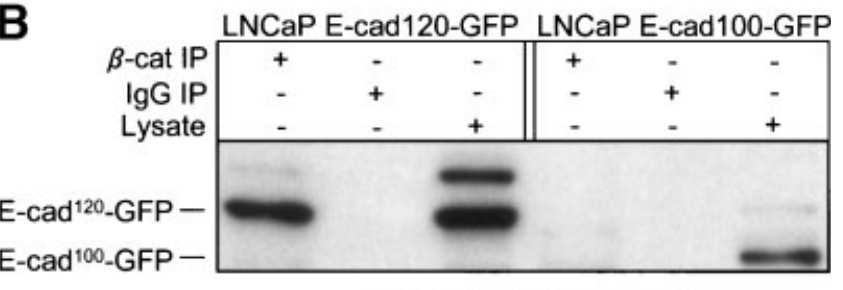

Western: E-cadherin

Fig. 4. E-cad ${ }^{100}$-GFP does not associate with $\beta$-catenin. LNCaPEcad $^{120}$-GFP cells or LNCaP E-cad ${ }^{100}$-GFP cells were immunoprecipitated with either an antibody to (A) GFPor (B) $\beta$-catenin. A negative control immunoprecipitation was also performed. Immune complexes were resolved by SDS-PAGE and immunoblotted with (A) a $\beta$-catenin antibody or (B) an E-cadherin antibody. 


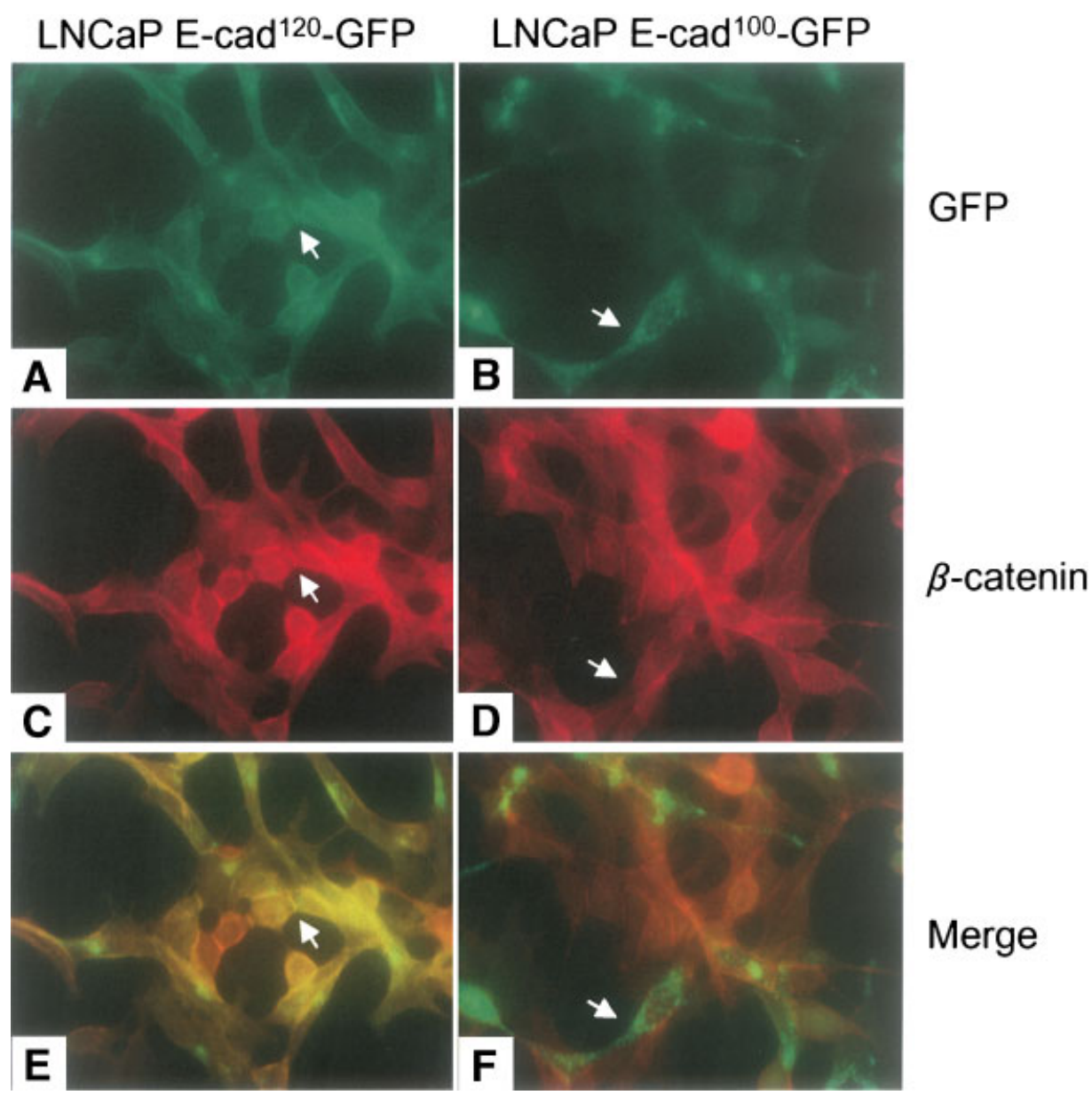

Fig. 5. E-cad ${ }^{100}$-GFP does not co-localize with $\beta$-catenin. Membrane localization (depicted by arrows) of E-cad ${ }^{120}-$ GFP $($ A) with endogenous $\beta$-catenin (C) is shown. Arrows depict lack of co-localization between E-cad ${ }^{100}$-GFP(B) and endogenous $\beta$-catenin (D). $\beta$-catenin was visualized using a C-terminal antibody. The merged images (E, F) demonstrate co-localization of $\beta$-catenin with E-cad ${ }^{120}$-GFP and not E-cad ${ }^{100}$-GFP. Magnification is at $60 \times$. [Color figure can be viewed in the online issue, which is available at $w w w$.interscience.wiley.com.]

and E). In contrast, E-cad ${ }^{100}$-GFP displayed a punctuate expression pattern (Fig. 5B). Expression of $\beta$-catenin remained localized to the membrane, presumably through association with endogenous E-cadherin (Fig. 5D). However, the merged image demonstrated that E-cad ${ }^{100}$-GFP does not co-localize with endogenous $\beta$-catenin, supporting the results obtained from the co-immunoprecipitation experiments (Fig. 5F).

\section{E-cad ${ }^{100}$ Downregulates Endogenous E-Cadherin Expression}

Previous studies have shown that overexpression of truncated cadherin constructs can affect the expression of endogenous cadherin protein [22-24]. We hypothesized that E-cad ${ }^{100}$ may function to alter the expression of endogenous E-cadherin in LNCaP cells. We utilized a C-terminal-specific E-cadherin antibody to distinguish between endogenous E-cadherin and E-cad ${ }^{100}$-GFP in immunofluorescence experiments.
In the LNCaP E-cad ${ }^{100}$ cell line, or in MCF-7 cells in which E-cad ${ }^{100}$ was transiently expressed, cells which were positive for E-cad ${ }^{100}$-GFP expression were found to have marked downregulation of endogenous membrane E-cadherin expression (Fig. 6A). Similarly, cells which lacked GFP expression had retained endogenous E-cadherin expression (Fig. 6A). The percentage of GFP positive cells that contained downregulated endogenous E-cadherin was over $80 \%$ in both the LNCaP E-cad ${ }^{100}$ cell line and in MCF-7 cells in which E-cad ${ }^{100}$-GFP was transiently expressed (Fig. 6A). To biochemically test whether endogenous membrane E-cadherin was being downregulated in E-cad ${ }^{100}$-GFP overexpressing cells, cell membrane extracts of LNCaP vector and LNCaP E-cad ${ }^{100}$-GFP cells were prepared and analyzed by Western blot for E-cadherin. Downregulation of endogenous E-cadherin was observed in $\mathrm{LNCaP}$ E-cad ${ }^{100}$-GFP cells compared to vector control (Fig. 6B). Densitometric analysis demonstrated a $40 \%$ reduction of endogenous E-cadherin expression 
A
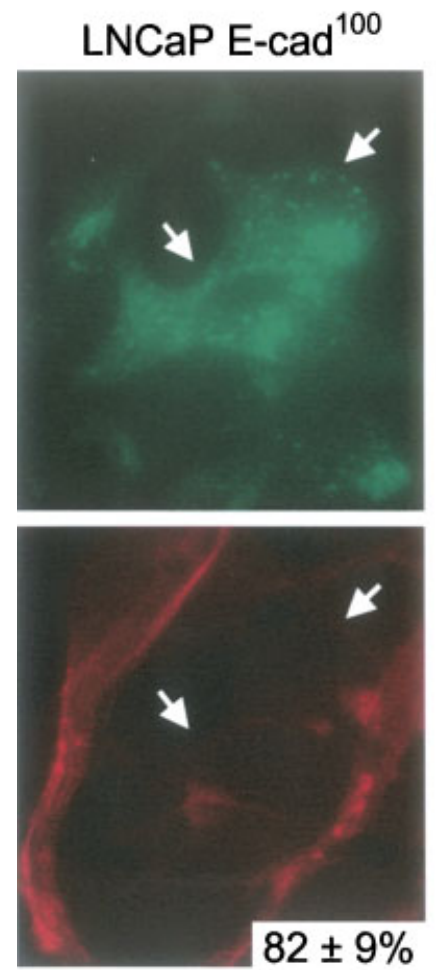
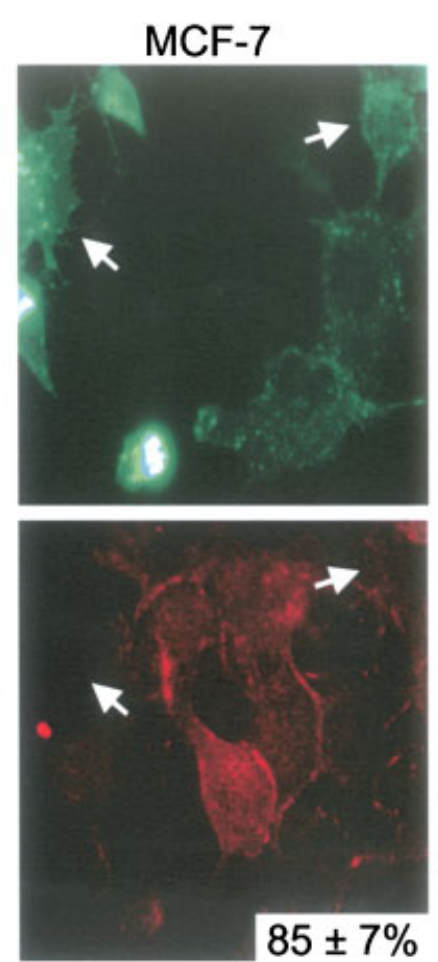

B
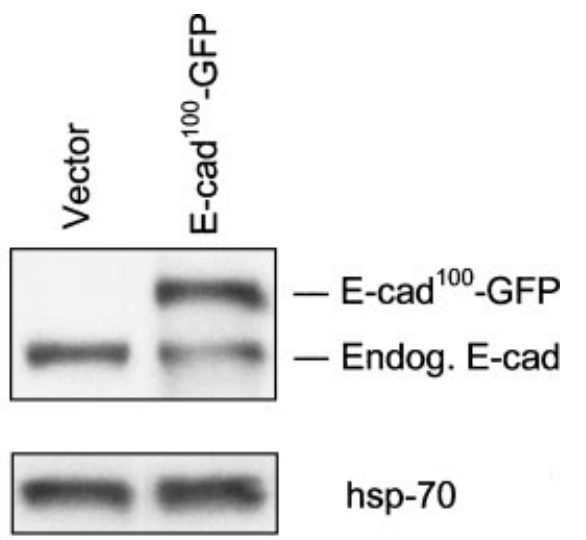

hsp-70

\section{C}

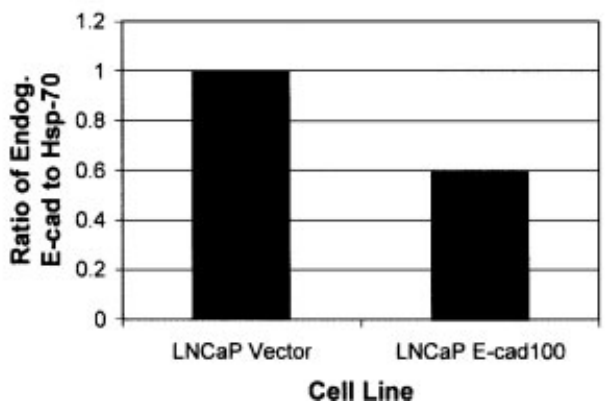

Fig. 6. E-cad ${ }^{100}$ overexpression downregulates endogenous E-cadherin. A: LNCaPE-cad ${ }^{100}$-GFP cells or MCF-7 cells transiently transfected with E-cad ${ }^{100}$-GFP were stained with a C-terminal E-cadherin antibody (bottom panels) and visualized for GFP (top panels). Arrows depict cells which express E-cad ${ }^{100}$-GFP and contain downregulated endogenous E-cadherin. Magnification is at $40 \times$. B: The membrane/ organelle fractions of untreated LNCaP vector and LNCap E-cad ${ }^{100}$-GFP cells were isolated, resolved by SDS-PAGE and immunoblotted for E-cadherin. The blot was re-probed for hsp-70 as a loading control.C:TheWestern blot in (B) was subjected to densitometric analysis. The ratio of endogenous E-cadherin to the hsp-70 loading control was compared between LNCaPE-cadI00-GFP cells and LNCaP vector cells. The result is representative of two experiments. [Color figure can be viewed in the online issue, which is available at www.interscience.wiley.com.]

in E-cad ${ }^{100}$-overexpressing cells compared to vector control (Fig. 6C). These data demonstrate that E-cad ${ }^{100}$ GFP overexpression downregulates endogenous membrane E-cadherin expression.

\section{Overexpression of E-cad ${ }^{100}$ Potentiates PKC-Induced Apoptosis}

We have previously elucidated a role for E-cadherin in aggregation-dependent survival of LNCaP cells, in which PKC-induced aggregation of LNCaP cells resulted in the E-cadherin-dependent survival of aggregated cells, and death of cells unable to aggregate [10]. We hypothesized, therefore, that downregulation of functional endogenous E-cadherin through overexpression of E-cad ${ }^{100}$ could potentially lead to an impaired ability of cells to aggregate and results in an inhibition of cell survival. To test this hypothesis, LNCaP E-cad ${ }^{100}$-GFP, LNCaP E-cad ${ }^{120}$-GFP, and LNCaP vector control cells were treated with TPA for $24 \mathrm{hr}$ and the viability of the aggregated cell population was measured in a trypan blue exclusion assay. TPA treatment resulted in the death of $24 \%$ in the control cells and $27 \%$ in E-cad ${ }^{120}$-GFP-overexpressing cells (Fig. 7). In contrast, TPA treatment resulted in the death of $38 \%$ of cells overexpressing E-cad ${ }^{100}$-GFP, which was statistically significant. These results demonstrate that E-cad ${ }^{100}$ overexpression in the aggregated LNCaP cell population potentiated cell death following PKC activation and suggested that E-cad ${ }^{100}$ can functionally inhibit cell survival.

\section{DISCUSSION}

We have demonstrated a role for a truncated E-cadherin species, which represents a physiologic, calpain-generated fragment of E-cadherin, in inhibiting aggregation-dependent survival in prostate epithelial cells. Co-localization and co-immunoprecipitation experiments confirmed that E-cad ${ }^{100}$-GFP did not associate with $\beta$-catenin. Fluorescence microscopy analysis 


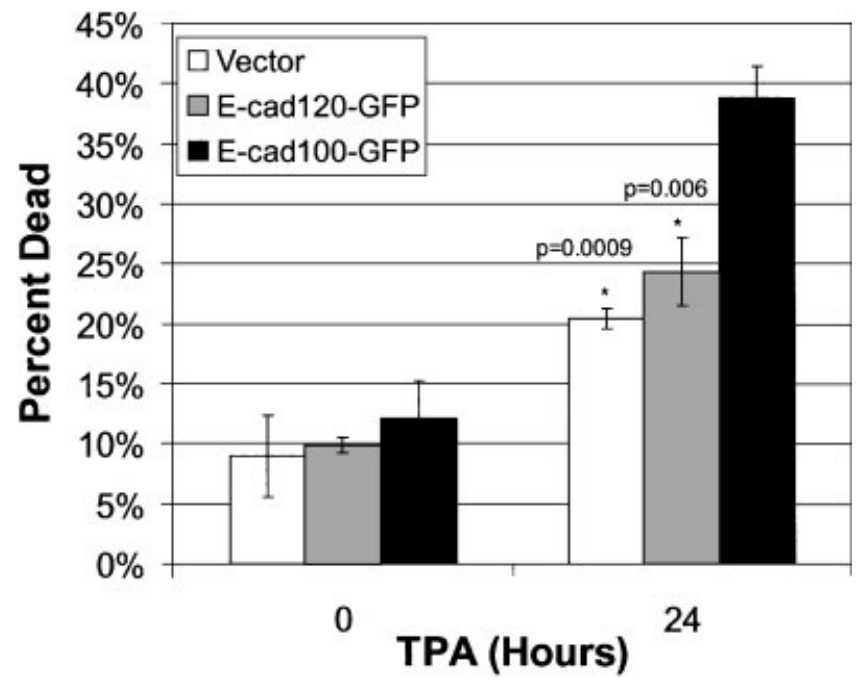

Fig. 7. $E-$ cad $^{100}$ overexpression potentiates PKC-induced cell death. LNCaP vector, LNCaP E-cad ${ }^{120}$-GFP, and LNCaP E-cad ${ }^{100}$ GFP cells were treated with TPA for $24 \mathrm{hr}$. The percentage of dead cells before and after treatment was determined. The experiment was performed in triplicate. The percentage of dead cells in the LNCaP E-cad ${ }^{100}$-GFP cell line was significantly higher than the TPA-treated control cell lines $(P<0.05)$.

revealed that E-cad ${ }^{100}$-GFP expression downregulated membrane E-cadherin expression, and functionally potentiated cell death. These results imply a role of E-cad ${ }^{100}$ in the regulation of LNCaP cell survival.

Cell-matrix and cell-cell contacts have been shown to propagate cellular survival signals $[4,10,25,26]$. The disruption of these contacts by intracellular proteases has been demonstrated. Caspases have been shown to cleave proteins in the focal adhesion complex, such as focal adhesion kinase and paxillin $[27,28]$. In addition, caspases have been shown to target E-cadherin and $\beta$-catenin in some apoptotic programs [15,29]. Despite an established role for caspases in apoptosis, there is evidence that calpain can also play a role in some apoptotic processes [30-32]. Calpain has also been shown to proteolytically cleave proteins in the focal adhesion complex, such as integrins, as well as cytoskeletal substrates during apoptosis [33-35]. Our data suggests that the cytoplasmic domain of E-cadherin, the main component of the adherens junction, is cleaved during PKC-induced apoptosis of LNCaP cells. We hypothesized that this cleavage, which removes the $\beta$-catenin binding domain, adversely affects cell survival.

To investigate the role of E-cad ${ }^{100}$ in cell survival, an E-cad ${ }^{100}$-GFP construct was engineered. Fluorescence microcopy revealed that the expression pattern of E-cad ${ }^{100}$-GFP was punctate in nature, compared to membranous staining for E-cad ${ }^{120}$-GFP. These results are similar to findings from a previous study, which demonstrated punctuate expression of overexpressed
E-cadherin mutants which contained truncations in the cytoplasmic domain [2]. The cytoplasmic localization of these mutants likely stems from the lack of association of these cadherins to the actin cytoskeleton. Subcellular fractionation of TPA-treated LNCaP lysates and cells expressing E-cad ${ }^{100}$-GFP demonstrated that both endogenous and exogenous $E-\mathrm{cad}^{100}$ resided exclusively in membrane biochemical compartments, which consist of plasma and organelle membranes (data not shown). We speculate then, that endogenous E-cad ${ }^{100}$ may be internalized following cleavage and be localized similarly to exogenous E-cad ${ }^{100}$. Alternatively, endogenous E-cad ${ }^{100}$ may retain association with the plasma membrane. The lack of an antibody that can specifically detect endogenous E-cad ${ }^{100}$ and not full-length E-cadherin prevents determination of its precise localization via immunofluorescence microscopy.

We have shown that overexpression of E-cad ${ }^{100}$ downregulates endogenous E-cadherin at the membrane and that this inhibits cell survival. One of the ways in which E-cadherin may promote cell survival is through association with growth factor signaling pathways. It has been shown that the engagement of E-cadherin at cell-cell contacts can activate both PI3 and MAP kinase pathways [36,37]. $\beta$-catenin links E-cadherin to the epidermal growth factor receptor, and has been shown to associate with the $\mathrm{p} 85$ subunit of PI3 kinase [38,39]. The removal of the $\beta$-catenin binding domain by calpain may therefore remove the association of E-cadherin with growth factor receptors and this might inhibit the survival of cells when challenged with death stimuli. Prior studies have shown that transfection of truncated cadherin molecules can affect the expression of endogenous cadherin protein $[22,23]$. We hypothesize that a reduction in membranous fulllength E-cadherin resulting from E-cad ${ }^{100}$ overexpression might result in a hindrance of cells to mediate cell survival signals upon receipt of cell death stimuli. Thus, we propose that cleavage of E-cadherin might inhibit cell survival in two ways: through the removal of the $\beta$-catenin binding domain of intact E-cadherin at the plasma membrane, and the separate effect of this fragment on downregulation of endogenous membrane E-cadherin.

The finding that E-cad ${ }^{100}$ inhibits membrane endogenous E-cadherin expression raised the question of the mechanism of how this occurs. One possibility is that E-cad ${ }^{100}$ binds to endogenous E-cadherin and sequesters it in the cytoplasm, preventing it from reaching the cell surface. It has been shown that E-cadherin can form cis-dimers on the membrane of cells [40]. However, no evidence has been reported of E-cadherin forming homodimers in the cytoplasm, and this is unlikely since E-cad ${ }^{100}$ and endogenous E-cadherin did not co-localize in this study. Another 
possibility is that the retention of E-cad ${ }^{100}$ in cytoplasmic compartments impairs the cell surface transport of both itself and endogenous E-cadherin. The exact mechanism of how this regulation occurs remains to be elucidated.

Many previous studies have examined the biological role of E-cadherin mutants which lack cytoplasmic domains and have demonstrated that this domain is required for normal cell adhesion [2,3,41]. Previous studies from our lab demonstrated a novel, calpain-dependent physiological pathway in which the $\beta$-catenin binding domain is removed [12]. We propose that the removal of this domain leads to disruption of cellular adhesion and can inhibit cellular survival following apoptotic stimuli.

\section{REFERENCES}

1. Yap AS, Brieher WM, Gumbiner BM. Molecular and functional analysis of cadherin-based adherens junctions. Annu Rev Cell Dev Biol 1997;13:119-146.

2. Nagafuchi A, Takeichi M. Cell binding function of E-cadherin is regulated by the cytoplasmic domain. Embo J 1988;7(12):36793684.

3. Ozawa M, Ringwald M, Kemler R. Uvomorulin-catenin complex formation is regulated by a specific domain in the cytoplasmic region of the cell adhesion molecule. Proc Natl Acad Sci USA 1990;87(11):4246-4250.

4. Kantak SS, Kramer RH. E-cadherin regulates anchorageindependent growth and survival in oral squamous cell carcinoma cells. J Biol Chem 1998;273(27):16953-16961.

5. Peluso JJ, Pappalardo A, Fernandez G. E-cadherin-mediated cell contact prevents apoptosis of spontaneously immortalized granulosa cells by regulating Akt kinase activity. Biol Reprod 2001;64(4):1183-1190.

6. Li G, Satyamoorthy K, Herlyn M. N-cadherin-mediated intercellular interactions promote survival and migration of melanoma cells. Cancer Res 2001;61(9):3819-3825.

7. Peluso JJ, Pappalardo A, Trolice MP. N-cadherin-mediated cell contact inhibits granulosa cell apoptosis in a progesteroneindependent manner. Endocrinology 1996;137(4):1196-1203.

8. Tran NL, Adams DG, Vaillancourt RR, Heimark RL. Signal transduction from $\mathrm{N}$-cadherin increases $\mathrm{Bcl}-2$. Regulation of the phosphatidylinositol 3-kinase/Akt pathway by homophilic adhesion and actin cytoskeletal organization. J Biol Chem 2002;277(36):32905-32914.

9. Erez N, Zamir E, Gour BJ, Blaschuk OW, Geiger B. Induction of apoptosis in cultured endothelial cells by a cadherin antagonist peptide: Involvement of fibroblast growth factor receptormediated signalling. Exp Cell Res 2004;294(2):366-378.

10. Day ML, Zhao X, Vallorosi CJ, Putzi M, Powell CT, Lin C, Day KC. E-cadherin mediates aggregation-dependent survival of prostate and mammary epithelial cells through the retinoblastoma cell cycle control pathway. J Biol Chem 1999;274(14):96569664.

11. Vallorosi CJ, Day KC, Zhao X, Rashid MG, Rubin MA, Johnson KR, Wheelack MJ, Day ML. Truncation of the beta-catenin binding domain of E-cadherin precedes epithelial apoptosis during prostate and mammary involution. J Biol Chem 2000; 275(5):3328-3334.
12. Rios-Doria J, Day KC, Kuefer R, Rashid MG, Chinnaiyan AM, Rubin MA, Day ML. The role of calpain in the proteolytic cleavage of E-cadherin in prostate and mammary epithelial cells. J Biol Chem 2003;278(2):1372-1379.

13. Wheelock MJ, Buck CA, Bechtol KB, Damsky CH. Soluble 80-kd fragment of cell-CAM 120/80 disrupts cell-cell adhesion. J Cell Biochem 1987;34(3):187-202.

14. Noe V, Fingleton B, Jacobs K, Crawford HC, Vermeulen S, Steelant W, Bruyneel E, Matrisian LM, Mareel M. Release of an invasion promoter E-cadherin fragment by matrilysin and stromelysin-1. J Cell Sci 2001;114(Pt 1):111-118.

15. Steinhusen U, Weiske J, Badock VV, Tauber R, Bommert K, Huber $\mathrm{O}$. Cleavage and shedding of E-cadherin after induction of apoptosis. J Biol Chem 2000;13:13.

16. Wang KK. Calpain and caspase: Can you tell the difference? Trends Neurosci 2000;23(1):20-26.

17. Nakagawa T, Yuan J. Cross-talk between two cysteine protease families. Activation of caspase- 12 by calpain in apoptosis. J Cell Biol 2000;150(4):887-894

18. Neumar RW, Xu YA, Gada H, Guttmann RP, Siman R. Cross-talk between calpain and caspase proteolytic systems during neuronal apoptosis. J Biol Chem 2003;278(16):14162-14167.

19. McCollum AT, Nasr P, Estus S. Calpain activates caspase-3 during UV-induced neuronal death but only calpain is necessary for death. J Neurochem 2002;82(5):1208-1220.

20. Day ML, Zhao X, Wu S, Swanson PE, Humphrey PA. Phorbol ester-induced apoptosis is accompanied by NGFI-A and c-fos activation in androgen-sensitive prostate cancer cells. Cell Growth Differ 1994;5(7):735-741.

21. Gutierrez AA, Arias JM, Garcia L, Mas-Oliva J, GuerreroHernandez A. Activation of $\mathrm{a} \mathrm{Ca}^{2+}$-permeable cation channel by two different inducers of apoptosis in a human prostatic cancer cell line. J Physiol 1999;517(Pt 1):95-107.

22. Levine $\mathrm{E}$, Lee $\mathrm{CH}$, Kintner C, Gumbiner BM. Selective disruption of E-cadherin function in early Xenopus embryos by a dominant negative mutant. Development 1994;120(4):901-909.

23. Navarro P, Ruco L, Dejana E. Differential localization of VE- and $\mathrm{N}$-cadherins in human endothelial cells: VE-cadherin competes with N-cadherin for junctional localization. J Cell Biol 1998; 140(6):1475-1484.

24. Fujimori T, Takeichi M. Disruption of epithelial cell-cell adhesion by exogenous expression of a mutated nonfunctional N-cadherin. Mol Biol Cell 1993;4(1):37-47.

25. Stupack DG, Cheresh DA. Get a ligand, get a life: Integrins, signaling and cell survival. J Cell Sci 2002;115(Pt 19):3729-3738.

26. Herren B, Levkau B, Raines EW, Ross R. Cleavage of beta-catenin and plakoglobin and shedding of VE-cadherin during endothelial apoptosis: Evidence for a role for caspases and metalloproteinases. Mol Biol Cell 1998;9(6):1589-1601.

27. Wen LP, FahrniJA, Troie S, Guan JL, Orth K, Rosen GD. Cleavage of focal adhesion kinase by caspases during apoptosis. J Biol Chem 1997;272(41):26056-26061.

28. Levkau B, Herren B, Koyama H, Ross R, Raines EW. Caspasemediated cleavage of focal adhesion kinase pp125FAK and disassembly of focal adhesions in human endothelial cell apoptosis. J Exp Med 1998;187(4):579-586.

29. Brancolini C, Lazarevic D, Rodriguez J, Schneider C. Dismantling cell-cell contacts during apoptosis is coupled to a caspasedependent proteolytic cleavage of beta-catenin. J Cell Biol 1997;139(3):759-771.

30. Squier MK, Cohen JJ. Calpain, an upstream regulator of thymocyte apoptosis. J Immunol 1997;158(8):3690-3697. 
31. Squier MK, Miller AC, Malkinson AM, Cohen JJ. Calpain activation in apoptosis. J Cell Physiol 1994;159(2):229-237.

32. Wood DE, Thomas A, Devi LA, Berman Y, Beavis RC, Reed JC, Newcomb EW. Bax cleavage is mediated by calpain during drug-induced apoptosis. Oncogene 1998;17(9):1069-1078.

33. Meredith J Jr, Mu Z, Saido T, Du X. Cleavage of the cytoplasmic domain of the integrin beta3 subunit during endothelial cell apoptosis. J Biol Chem 1998;273(31):19525-19531.

34. Nath R, Raser KJ, Stafford D, Hajimohammadreza I, Posner A, Allen H, Talanian RV, Yven P, Gilbertsen RB, Wang KK. Nonerythroid alpha-spectrin breakdown by calpain and interleukin 1 beta-converting-enzyme-like protease(s) in apoptotic cells: Contributory roles of both protease families in neuronal apoptosis. Biochem J 1996;319(Pt 3):683-690.

35. Carragher NO, Fincham VJ, Riley D, Frame MC. Cleavage of focal adhesion kinase by different proteases during SRCregulated transformation and apoptosis. Distinct roles for calpain and caspases. J Biol Chem 2001;276(6):4270-4275.

36. Pece S, Chiariello M, Murga C, Gutkind JS. Activation of the protein kinase Akt/PKB by the formation of E-cadherinmediated cell-cell junctions. Evidence for the association of phosphatidylinositol 3-kinase with the E-cadherin adhesion complex. J Biol Chem 1999;274(27):19347-19351.

37. Pece S, Gutkind JS. Signaling from E-cadherins to the MAPK pathway by the recruitment and activation of epidermal growth factor receptors upon cell-cell contact formation. J Biol Chem 2000;275(52):41227-41233.

38. Hoschuetzky H, Aberle H, Kemler R. Beta-catenin mediates the interaction of the cadherin-catenin complex with epidermal growth factor receptor. J Cell Biol 1994;127(5):1375-1380.

39. Woodfield RJ, Hodgkin MN, Akhtar N, Morse MA, Fuller KJ, Saqib K, Thompson NT, Wakelam MJ. The p85 subunit of phosphoinositide 3-kinase is associated with beta-catenin in the cadherin-based adhesion complex. Biochem J 2001;360(Pt 2): 335-344.

40. Takeda H, Shimoyama Y, Nagafuchi A, Hirohashi S. E-cadherin functions as a cis-dimer at the cell-cell adhesive interface in vivo. Nat Struct Biol 1999;6(4):310-312.

41. Nagafuchi A, Takeichi M. Transmembrane control of cadherinmediated cell adhesion: A $94 \mathrm{kDa}$ protein functionally associated with a specific region of the cytoplasmic domain of E-cadherin. Cell Regul 1989;1(1):37-44. 